only say what is likely to be true based on the volume and quality of evidence available. Poor research integrity misleads doctors, meaning what we tell our patients might simply be wrong. In 2001 a single misreported trial, study 329, led to millions of adolescents being prescribed an antidepressant that didn't work, and caused suicidal behaviour. This was entirely avoidable. I believe there are three fixes to such problems in evidence-based medicine: transparency; strategic campaigning; and education. Transparency so we know about missing data and poor methodology Half of all clinical trial results are never reported, and positive trials are twice as likely to be published as negative trials. This means we often don't learn about harms data, and the data we have are biased. Hidden documents mean we don't know when publication bias is happening: readers accept biased results, because they trust journals and authors to maintain reporting integrity. Transparent reporting fixes publication bias, allowing us to see the most accurate picture of the evidence available. Transparent trial registration, protocols, and editorial practices allow us to detect poor methodology and interpret the evidence accordingly. Such transparency has very real consequences: If all data had been transparently reported, study 329 would have shown the lack of efficacy and increases in harm of paroxetine, and thousands of lives may have been saved. Strategic campaigning to effect real-world change Outcome switching and publication bias remain prevalent, showing decades of prevalence studies do not in themselves solve these problems. Guardians of evidence-based medicine must be more strategic in fixing its flaws. There are strong financial, academic and personal incentives for trialists to comply with requests from journal editors. Appealing to editors to require full registration and transparent reporting is therefore likely to motivate trial authors in good practice. Incentives such as CONSORT and ICMJE do just this and provide a standard to which journals can be held accountable. Funding is arguably the biggest barrier to research. Plan S sets out 10 principles to improve research funded by public grants, including funders' research integrity requirements. Future projects should learn from this: researchers are likely to comply with such requirements to secure funding. Education so people know what to do Responses to correction letters in the COMPare project showed widespread misunderstandings around the reporting of trial outcomes. Education is needed to show doctors and patients what good trial reporting looks like, so they can make informed clinical decisions with the evidence available. In COMPare we did this through journal correspondence, leading to policy changes and extensive discussion in the clinical trials community. Education should be considered more broadly: teaching EBM at schools sets standards for the next generation of leaders; educating the public creates appropriate intolerance to poor research practice; and open discussion of existing research educates doctors and patients about poor research practice affecting clinical decisions.

\section{WE NEED LESS RESEARCH, BETTER RESEARCH, AND RESEARCH DONE FOR THE RIGHT REASONS}

Tom Roberts. Royal College of Emergency Medicine, London, UK

10.1136/bmjebm-2019-EBMLive.76

The Trainee Emergency Research Network (TERN), funded by the Royal College of Emergency Medicine (RCEM), is a new initiative that aims to demystify research and increase research engagement amongst Emergency Medicine (EM) clinicians. It was launched in 2018 and is ideally placed to improve how EM research is planned and conducted. Whilst the value of evidence-based medicine in Emergency Departments (EDs) is recognised, the unique pressures of the ED setting makes conducting research and disseminating good practice particularly challenging. TERN was designed to tackle these challenges with a focus on three important pillars to engage busy EM Clinicians: 1) Answer practice-changing questions 2) Robust and achievable data collection 3) Recognition Point 1, the research question has to be important and applicable to a trainees' practice, both to encourage engagement and create impact. Point 2, the research has to be designed rigorously so that the data collection is clear and achievable within EDs and can be translated into clinical practice. Point 3, trainee contributions have to be recognised throughout. We will choose research questions that mirror the 2017 James-Lind/RCEM research priority setting partnership. This will allow TERN to frame its research questions around themes that have been recognised as vital in EM. TERN's strength lies in accessing multiple ED sites for standardised data collection, ideally over short collection periods, to obtain nationally representative snapshots of patients and practice. This enables, for example, small pilot studies and subsequent multi-site prospective observational cohort studies to be conducted rapidly. We recognise that collecting data in multiple sites leads to potential issues surrounding data monitoring and governance. Simplifying the research design of studies by only collecting data that genuinely adds to the research question, will support the collection of accurate data. TERN will also harness the use of online data collection, which allows for live data monitoring and a clear audit trail of all data entered. This will allow busy ED clinicians to concentrate on data collection and allow the study team to have clear oversight of the project at local, regional and national levels. TERN is new but the response from the EM community, both academic and non-academic, has been very encouraging. Thanks to this support, within 8 months, we already have multiple successes, including our first primary research project, TIRED, which has 111 UK and Ireland sites signed up for data collection. We believe that by giving EM clinicians the opportunity to engage in high quality projects and contribute to a national data collection process, we can move away from the current model of EM evidence generation that typically relies on collections of small, often poorlydesigned studies with limited compatibility. With our work, we aim to be transparent and seek guidance throughout our research designs, to ensure our projects stand up to the highest of research and statistical standards. Part of this process is opening dialogues and 'EBM Live' is the perfect forum to start this.

\section{AUTOMATING THE PROCESS OF SYSTEMATIC REVIEWS IN HEALTHCARE RESEARCH - A METHODOLOGICAL SYSTEMATIC REVIEW}

${ }^{1,2,3}$ Razia Aliani, ${ }^{1}$ Nicole Pitcher, ${ }^{1}$ Isabelle Boutron. ${ }^{1}$ Cochrane, Paris, France; ${ }^{2}$ École des hautes études en santé publique (EHESP), Rennes, France; ${ }^{3}$ University of Sheffield, Sheffield, UK

\subsection{6/bmjebm-2019-EBMLive.77}

Objectives Systematic Reviews (SRs) are the cornerstone of evidence-informed healthcare decision making. However, they are extremely resource-intensive and commonly take 2 to 3 years to complete. One of the solutions put forward to support 
reviewers and reduce the time required to conduct SRs is automation.

With recent advancements in artificial intelligence and machine learning, many tools have been, and are currently being, developed to support different stages of SR process. To date, the range of automated tools available, and their effectiveness, is unclear. To remedy this gap, we have undertaken a methodological systematic review.

The specific objectives of this methodological SR are to:

- Classify existing tools according to the automation approach, the stage of SR supported and the level of automation.

- Compare the available tools according to each stage that they support and identify their strengths and limitations.

- Determine the effectiveness of the tools.

- Present and appraise evaluations of automated tools.

Method This review is based on the Preferred Reporting Items for Systematic review and Meta-Analyses (PRISMA-P) Guidelines.

We reviewed all published articles, grey literature, reports and software manuals that evaluate automated and semi-automated tools that support healthcare-related SRs, from screening to write-up. Only tools that are fully developed were eligible for inclusion.

The systematic review toolbox (SR ToolBox), PubMed, Google and Google were systematically searched. Titles, abstracts and full articles were reviewed for inclusion independently by two reviewers. Data extraction and quality appraisal were undertaken independently by two reviewers, with disagreements resolved by consensus or by arbitration by a third reviewer if necessary.

Tools' characteristics and performance metrics reported in the included studies were extracted and tabulated. To enable comparisons, tools were grouped according to stages of SR they support, and the type of algorithms deployed in them.

Results This review is currently in the data extraction stage and will be completed by the first week of June 2019 .

This presentation will focus on the evidence available on automated tools that support the screening, data extraction, quality appraisal and write-up phases of SRs. For each phase, we will present data on the number of tools that have been developed and the number of studies that have evaluated them. We will discuss the strengths and limitations of the methods and standards used to evaluate existing tools, and implications for future research and SR practice.

Conclusions This review constitutes an important step in easing the transition of SR production from a primarily manual process to a semi-automated one. It will inform current collaborative efforts aimed at the development of evidenceinformed integrative automated systems for conducting high quality SRs in healthcare research.

\section{SUSTAINING AND DEVELOPING MOVEMENTS, LIKE EVIDENCE LIVE, IN MEDICINE: LESSONS TO BE LEARNT WHEN DOES A CAMPAIGN BECOME A MOVEMENT - AND HOW DO WE IMPLEMENT A MOVEMENT INTO PRACTICE?}

\footnotetext{
${ }^{1,2}$ Neil Chanchlani. ${ }^{1}$ Royal Devon and Exeter Hospital, Exeter, UK; ${ }^{2}$ Canadian Medical Association, Ottawa, Canada
}

Social movements across medicine, research, and publishing, such as EBM Live, Choosing Wisely, and Reducing Research Waste are well-intentioned, but are met with varying degrees of success. We need to learn from recent successful social movements that have reached international circulation and had fundamental impact, such as \#MeToo and Black Lives Matter. The EBM manifesto's aim to reduce bias, wastage, error fraud research is driven by a small community of researchers, academics, and publishers, largely from the United Kingdom. What percentage of end users, including researchers, patients, and public, are engaged or even aware of the movement? We must move away from simply raising awareness amongst small communities to co-producing and collaborating with them. Looking at successful social movements within and outside medicine that have generated transformational change and inspired the public, the following themes emerge:

1. Have a clear purpose and vision for the movement. This has to be simple, short, and direct rather than a complicated and detailed plan. This will hook and unify the targeted audience and break down interdisciplinary barriers. (Satell, Harvard Business Review 2016).

2. Use a structured framework to ensure standardisation across the movement. In order to enact change locally, nationally, and internationally, there needs to be clear guidance. For example, the Knowledge-to-Action Cycle, generated by Canadian Institute of Health Research, can be used as a guiding framework to increase the relevance, applicability, and impact of research findings (Field, Implementation Science 2014). This will increase clarity and process within the movement.

3. Training nominated champions will increase uptake of the movement. Nominated champions should undergo sponsored training which will empower them to create a shared vision amongst their teams within hospital, university, or organisation. Formal accreditation, such as the Knowledge Translation Professional Certificate ${ }^{\mathrm{TM}}$, or informal opportunities, such as workshops and summer courses run by Evidence Live are a starting point. Cementing awareness can be formalised through the National Institute for Health Research Good Clinical Practice module and local induction for all healthcare and university staff, and not just those involved in research. This will ensure everyone is involved and engaged in the movement.

4. Continuous monitoring and evaluation are necessary for a successful movement. An iterative process will allow strategy review, monitoring of activities, and amendments to be made in line with the movement's values. Sustaining a movement in medicine is difficult. For real change to occur, the whole community needs to be engaged. Evidence Live is an inspiring conference with an ambitious manifesto, ultimately aiming to improve patient care. Unfortunately, we are still failing to reach the majority of the medical community. To do this, we need to learn from other social movements to understand their roadmaps and how to improve ours. This will enable movements like Evidence Live to be successfully implemented into practice.

\section{MISREPRESENTED OR UNVERIFIED AFFILIATIONS IN THE LITERATURE SHOULD BE ADDRESSED BY A CONSENSUS ON THE DEFINITION OF AFFILIATION}

Francisco Uribe, Vivienne Bachelet. Facultad de Ciencias Médicas, Universidad de Santiago de Chile, Santiago, Chile 